Received: 06.06.2021

Revised: 06.08.2021

Accepted: 27.08 .2021

DOI: $10.17804 / 2410-9908.2021 .4 .023-033$

\title{
THE INFLUENCE OF 2 AT. \% Si ADDITION ON THE ANNEALING OF RADIATION-INDUCED DEFECTS IN THE Fe-13Cr ALLOY
}

\author{
A. L. Nikolaev \\ M.N. Miheev Institute of Metal Physics, Ural Branch of the Russian Academy of Sciences, \\ 18 S. Kovalevskoy St. Ekaterinburg, 620108, Russia \\ https://orcid.org/0000-0002-0858-9820 nikolaev@imp.uran.ru \\ *Corresponding author: E-mail: nikolaev@imp.uran.ru \\ Address for correspondence: 18, S. ul. Kovalevskoy, Ekaterinburg, 620108, Ekaterinburg, Russian Federation \\ Tel.: +7 (343) 3783850
}

The paper presents data on electrical resistivity recovery in the $\mathrm{Fe}-13.4 \mathrm{Cr}$ and $\mathrm{Fe} 13.6 \mathrm{Cr}$ 1.9Si alloys during isochronal annealing after $5 \mathrm{MeV}$ electron irradiation below $77 \mathrm{~K}$. Long-range migration of radiation-induced defects starts slightly above $200 \mathrm{~K}$ in $\mathrm{Fe}-13.4 \mathrm{Cr}$. The silicon addition in Fe13.6Cr-1.9Si leads to immobilization of Frenkel pair defects thus making the peaks of the stages of the onset of long-range migration shift towards high temperatures up to $370 \mathrm{~K}$ and $420 \mathrm{~K}$ for self-interstitial atoms and vacancies, respectively. This finding confirms the data obtained earlier for Fe16Cr-Si alloys by means of positron annihilation technique (JNM 508(2018) 100-106) on trapping of radiation-induced defects on $\mathrm{Si}$ agglomerates (clusters consisting of several silicon atoms) formed during defect migration.

Keywords: Fe-Cr alloys, electron irradiation, radiation-induced defects, resistivity recovery, longrange defect migration, defect immobilization by Si agglomerates.

\section{Acknowledgment}

The research was carried out under the state assignment from the Ministry of Science and Higher Education of the Russian Federation (theme Function, No. AAAA-A19-119012990095-0). The author thanks Dr. D.A. Perminov for critically reading the manuscript.

\section{References}

1. Druzhkov A.P., Nikolaev A.L. Effect of Si concentration on formation of vacancy complexes in electron-irradiated Fe16Cr-Si alloys studied by positron annihilation. J. Nucl. Mater., 2018, vol. 508, pp. 100-106. DOI: 10.1016/j.jnucmat.2018.05.040.

2. Nikolaev A.L. Specificity of stage III in electron-irradiated Fe-Cr alloys. Phil. Mag., 2007, vol. 87, No. 31, pp. 4847-4874. DOI: 10.1080/14786430701468977.

3. Nikolaev A.L. Difference approach to the analysis of resistivity recovery data for irradiated short-range ordered alloys. Phil. Mag., 2009, vol. 89, No. 12, pp. 1017-1033. DOI: $10.1080 / 14786430902835651$.

4. Nikolaev A.L. Recovery of electrical resistivity, short-range order formation and migration of defects in electron-irradiated Fe-4Cr alloy doped with carbon. Phil. Mag., 2011, vol. 91, No. 6, pp. 879-898. DOI: 10.1080/14786435.2010.534740.

5. Druzhkov A.P., Nikolaev A.L. Effects of solute atoms on evolution of vacancy defects in electron-irradiated Fe-Cr-based alloys. J. Nucl. Mater., 2011, vol. 408 (2), pp. 194-200. DOI: 10.1016/j.jnucmat.2010.11.036. 
6. Dimitrov C., Dimitrov O. Composition dependence of defect properties in electron-irradiated Fe-Cr-Ni solid solutions. J. Phys. F: Met. Phys., 1984, vol. 14 (4), pp. 793-811. DOI: 10.1088/03054608/14/4/005.

7. Benkaddour A., Dimitrov C., Dimitrov O. Irradiation-induced defects in ferritic Fe-Cr alloys. Mater. Sci. Forum, 1987, vol. 15-18, pp. 1263-1268. DOI: 10.4028/www.scientific.net/MSF.15-18.1263.

8. Kohl W., Scheffel R., Heidsiek H., Lucke K. Investigation of the kinetics of short-range order formation and quenched-in vacancy annihilation in Au-15 at. \% Ag by resistivity measurements. Acta Metall., 1983, vol. 31, No. 11, pp. 1895-1908. DOI: 10.1016/0001-6160(83)90135-9.

9. Vaessen P., Lengeler B., Shilling W. Recovery of electrical resistivity in electronirradiated concentrated silver-zinc alloys. Rad. Effects, 1984, vol. 81 (3-4), pp. 277-292. DOI: $10.1080 / 00337578408206075$.

10. Mirebeau I., Hennion M., Parette G. First measurement of short-range-order inversion as a function of concentration in a transition alloy. Phys. Rev. Lett., 1984, vol. 53, pp. 687-690. DOI: 10.1103/PhysRevLett.53.687.

11. Apostolopoulos G., Lukianova V., Kotsina Z., Lagoyannis A., Mergia K., Harissopoulos S., Messoloras S. The influence of carbon on the resistivity recovery of proton irradiated $\mathrm{Fe}-11$ at. \% $\mathrm{Cr}$ alloys. Nuclear Materials and Energy, 2016, vol. 9, pp. 465-470. DOI: 10.1016/j.nme.2016.09.007.

12. Begoña Gómez-Ferrer. Resistivity recovery in $\mathrm{Fe}$ and $\mathrm{FeCr}$ alloys, SpringerBriefs in Applied Sciences and Technology, 2016.

13. Nikolaev A.L., Arbuzov V.L., Davletshin A.E. On the effect of impurities on resistivity recovery, short-range ordering, and defect migration in electron-irradiated concentrated $\mathrm{Fe}-\mathrm{Cr}$ alloys. J. Phys.: Condens. Matter., 1997, vol. 9, pp. 4385-4402. DOI: 10.1088/0953-8984/9/21/006.

14. Maury F., Lucasson A., Lucasson P., Moser P., Loreaux Y. Interstitial migration in dilute Fe-Si and Fe-Au alloys. J. Phys. F: Met. Phys., 1985, vol. 15, iss. 7, pp. 1465-1484. DOI:10.1088/0305-4608/15/7/007.

15. Takaki S., Fuss J., Kugler H., Dedek U., Schultz H. The resistivity recovery of high purity and carbon doped iron following low temperature electron irradiation. Rad. Effects, 1983, vol. 79 (3-4), pp. 87-122. DOI: 10.1080/00337578308207398. 
Подана в журнал: 06.06.2021

УДК 539.12.043:620.192.63

DOI: $10.17804 / 2410-9908.2021 .4 .023-033$

\title{
ВЛИЯНИЕ 2 АТ. \% Si НА ОТЖИГ РАДИАЦИОННЫХ ДЕФЕКТОВ В СПЛАВЕ $\mathrm{Fe}-13 \mathrm{Cr}$
}

\author{
А. Л. Николаев \\ Федеральное государственное бюджетное учреждение науки \\ Институт физики металлов им. М.Н. Михеева Уральского отделения Российской академии наук, \\ д. 18, ул. С. Ковалевской, Екатеринбург, Российская Федераџия \\ iD https://orcid.org/0000-0002-0858-9820 nikolaev@imp.uran.ru \\ *Ответственный автор. Электронная почта: nikolaev@imp.uran.ru \\ Адрес для переписки: ул. С. Ковалевской, 18, Екатеринбург, 620108, Российская Федерация \\ Тел.: +7 (343) 378-38-50
}

Представлены данные по возврату остаточного электросопротивления в сплавах $\mathrm{Fe}-13,4 \mathrm{Cr}$ и $\mathrm{Fe} 13,6 \mathrm{Cr}-1,9 \mathrm{Si}$ при изохронном отжиге после облучения электронами 5 МэВ ниже 77 К. В сплаве $\mathrm{Fe}-13,4 \mathrm{Cr}$ дальняя миграция радиационных дефектов начинается немного выше 200 К. В сплаве Fe13,6Cr-1,9Si добавка кремния приводит к иммобилизации дефектов Френкеля, в результате чего пики стадий начала дальней миграции собственных междоузельных и вакансий сдвигаются в сторону высоких температур до 370 К и 420 К соответственно. Эти данные подтверждают результаты, полученные ранее в сплавах $\mathrm{Fe} 16 \mathrm{Cr}-\mathrm{Si}$ методом аннигиляции позитронов (JNM 508(2018) 100-106), о захвате радиационных дефектов на кремниевых агрегатах (кластерах, состоящих из нескольких атомов кремния), формирующихся в процессе миграции дефектов.

Ключевые слова: сплавы $\mathrm{Fe}-\mathrm{Cr}$, облучение электронами, радиационные дефекты, возврат остаточного электросопротивления, дальняя миграция дефектов, иммобилизация дефектов агломератами из атомов $\mathrm{Si}$.

\section{1. Введение}

Сплавы $\mathrm{Fe}-\mathrm{Cr}$ являются основой для ферритных и ферритно-мартенситных сталей, используемых как конструкционный материал в атомных реакторах. Добавка 1,5-2 ат. \% Si улучшает коррозионную стойкость этих сталей в свинцово-висмутовой эвтектике, которая рассматривается в качестве перспективного теплоносителя в реакторах на быстрых нейтронах. Поэтому важно понимать, как добавка кремния влияет на радиационную повреждаемость этих сталей. Первым шагом в этом направлении является понимание, как подобная добавка влияет на свойства радиационных дефектов.

Ранее [1] были представлены данные по взаимодействию вакансий с атомами $\mathrm{Si}$ в матрице $\mathrm{Fe}-16$ ат. \% $\mathrm{Cr}$, легированной разным количеством кремния $(0,2 ; 0,75$ и 1,5 ат. \%) после облучения электронами с энергией 5 МэВ вблизи $300 \mathrm{~K}$, полученные методом аннигиляции позитронов, а именно, угловой корреляции аннигиляционного излучения.

Полученные результаты показывают, что изолированные атомы Si в разбавленном сплаве Fe16Cr-0,2Si не влияют на накопление и кластеризацию вакансий по сравнению с накоплением и кластеризацией в нелегированном сплаве $\mathrm{Fe}-16 \mathrm{Cr}$. Поэтому был сделан вывод, что они не захватывают мигрирующие вакансии и собственные междоузельные атомы (СМА) при $300 \mathrm{~K}$.

В отличие от $\mathrm{Fe}-16 \mathrm{Cr}$ и $\mathrm{Fe} 16 \mathrm{Cr}-0,2 \mathrm{Si}$, где под облучением при комнатной температуре происходит накопление кластеров вакансий, в сплаве с наивысшей концентрацией $\mathrm{Si}$, 
(Fe16Cr-1,5Si), происходит накопление моновакансий. Данные по аннигиляции позитронов указывают, что эти моновакансии декорированы атомами кремния, т.е. рядом с каждой моновакансией находится кластер из нескольких атомов кремния. Был сделан вывод, что под облучением при концентрации кремния выше 0,2 ат. \% происходит формирование кремниевых агрегатов (т. е. кластеров, состоящих из нескольких атомов $\mathrm{Si})^{1}$, которые захватывают и удерживают мигрирующие вакансии (т. е. осуществляют иммобилизацию вакансий) и не дают им объединяться в кластеры.

При ступенчатом изохронном отжиге после облучения в сплавах $\mathrm{Fe} 16 \mathrm{Cr}-0,75 \mathrm{Si}$ и $\mathrm{Fe} 16 \mathrm{Cr}-1,5 \mathrm{Si}$ наблюдали две стадии возврата: при 370 К и 420 К. Эти стадии были интерпретированы как диссоциация (освобождение с последующей миграцией) CMA (370 К) и вакансий $(420 \mathrm{~K})$, захваченных на кремниевых агрегатах в процессе облучения. Таким образом, согласно нашей интерпретации данных по аннигиляции позитронов при облучении вблизи комнатной температуры при концентрации 0,75 ат. \% Si и выше благодаря взаимодействию радиационных дефектов с атомами Si происходит формирование кремниевых агрегатов. Эти агрегаты захватывают оба типа дефектов из пары Френкеля ${ }^{2}$ (дефекты Френкеля - ДФ), мигрирующих при облучении вблизи 300 К, и удерживают их до достаточно высоких температур.

$\mathrm{B}$ нелегированных сплавах $\mathrm{Fe}-\mathrm{Cr}$ дальняя миграция Д $\Phi^{3}$ начинается (по крайней мере один из ДФ становится подвижным) при температурах 175-190 К [2-4]. Поэтому захват и удержание ДФ до высоких температур (370 и 420 К) при легировании примесью выглядят весьма необычно.

Чтобы получить независимое подтверждение этого факта с помощью другой методики, мы исследовали возврат остаточного электросопротивления (ОЭС) в сплавах Fe-13,4 ат. \% $\mathrm{Cr}(\mathrm{Fe}-13,4 \mathrm{Cr})$ и $\mathrm{Fe}-13,6$ ат. \% $\mathrm{Cr}+1,9$ ат. \% $\mathrm{Si}(\mathrm{Fe} 13,6 \mathrm{Cr}-1,9 \mathrm{Si})$, облученных электронами с энергией 5 МэВ при низких температурах. В отличие от [1], где исследованы модельные высокочистые сплавы, в настоящей работе для исследования использованы сплавы технической чистоты, которые по своему составу ближе к коммерческим сталям.

\section{2. Методика и характеристики образцов}

Оба сплава готовили дуговым переплавом порошков карбонильного железа, электролитического хрома и полупроводникового кремния в Институте металлургии им. Байкова (Москва) в алундовых тиглях слитками по 10 кг в атмосфере очищенного аргона. Затем слитки с промежуточными отжигами и травлением раскатывали в полосу толщиной 1 мм и в таком виде поставляли в Институт физики металлов. Здесь полосу вхолодную прокатывали в фольгу толщиной $0,1-0,12$ мм. Затем из фольги электроэрозионным способом вырезали четырехконтактные образцы с размерами измерительной части $4 \times 1,5 \mathrm{mм}^{2}$, которые электролитическим травлением утончали до 0.05 мм и отжигали 4 ч в безмасляном вакууме $10^{-4}$ Па при $\sim 1070$ К. Стандартным химическим анализом определяли содержание примесей замещения: основная примесь $\mathrm{Ni}-100$ appm. Содержание примесей внедрения определяли методом ядерного микроанализа: основная примесь С - 500 appm. Более подробные детали в [5].

Образцы сплавов облучали электронами 5 МэВ при температуре ниже 77 К. После облучения образцы перегружали в измерительный криостат и подвергали изохронному ступенчатому отжигу (с постепенным повышением температуры на каждой ступени и измерением ОЭС между ступенями) с температурным шагом 7,5-10 К и выдержкой на каждой ступени 7,5-10 мин соответственно.

\footnotetext{
${ }^{1}$ Чтобы избежать путаницы с кластерами дефектов, мы используем термин «агрегат».

${ }^{2}$ При электронном облучении дефекты генерируются в виде пар Френкеля, состоящих из СМА и вакансии.

3 Дальней миграцией называется миграция дефектов на расстояния, разделяющие дефекты из различных пар Френкеля и выше.
}

Nikolaev A. L. The influence of 2 at. \% Si addition on the annealing of radiation-induced defects in the Fe-13Cr alloy // Diagnostics, Resource and Mechanics of materials and structures. - 2021. - Iss. 4. - P. 23-33. - DOI: 10.17804/24109908.2021.4.023-033. 
По результатам строили и анализировали две зависимости от температуры отжига: возврат ОЭС $R=\Delta \rho(T) / \Delta \rho_{i r r}$ и скорость возврата ОЭС $-d R / d T=-\left(\Delta \rho_{i r r}\right)^{-1} d(\Delta \rho(T)) / d T$, где $\Delta \rho_{i r r}$ и $\Delta \rho(T)$ являются начальными (после облучения) и текущими (после отжига при температуре $T$ ) приростами ОЭС. Зависимость скорости возврата ОЭС от температуры $(-d R(T) / d T)$ также известна как спектр возврата ОЭС. Методики облучения, отжига образцов после облучения и измерения ОЭС подробно описаны в [2-4].

Для анализа поведения дефектов в концентрированных сплавах при отжиге после низкотемпературного облучения часто сравнивают кривые возврата ОЭС $R(T)$ в одинаковых образцах при различных начальных приростах ОЭС $\Delta \rho_{i r r}$, или, другими словами, начальных концентрациях ДФ $[3,6]$. Поэтому наши образцы также облучали до различных значений приростов ОЭС.

Три образца сплава Fe13,Cr-1,9Si облучали и отжигали в трех различных экспериментах. Два образца $\mathrm{Fe}-13,4 \mathrm{Cr}$ облучали до различных приростов ОЭС и отжигали в рамках одного эксперимента, как описано в [2]. Некоторые образцы Fe13.6Cr-1,9Si при переносе из устройства для облучения в измерительный криостат были перегреты до $\sim 100-110 \mathrm{~K}$. Поэтому для данных по возврату ОЭС $\left(R=\Delta \rho(T) / \Delta \rho_{i r r}\right)$ использовали нормировку на прирост ОЭС после отжига при $120 \mathrm{~K}$, т. е. $\Delta \rho_{i r r}=\Delta \rho_{120 K}$. Использование такой нормировки является корректным, так как, по крайней мере до $200 \mathrm{~K}$, зависимости $R(T)$ в обоих сплавах практически не зависят от значений $\Delta \rho_{i r r}$. Из сравнения с данными других авторов в близких сплавах [7] можно оценить, что $\Delta \rho_{120 к}$ в обоих сплавах составляет $\sim 55 \%$ от прироста ОЭС после облучения при 21 К. Поэтому использование $\Delta \rho_{120 K}$ вместо $\Delta \rho_{i r r}$ не влияет на характер полученных зависимостей и ведет к простой перенормировке значений возврата и скорости возврата ОЭС.

Из величины прироста ОЭС $\Delta \rho_{\text {irr }}$ легко рассчитать исходную концентрацию пар Френкеля $C_{F}^{0}$, если известен удельный вклад пар Френкеля в ОЭС $\rho_{F}, C_{F}^{0}=\Delta \rho_{i r r} / \rho_{F}$. Исходные концентрации ДФ при 120 К в образцах $\mathrm{Fe}-13,4 \mathrm{Cr}$ составляли 60 и 25 appm, принимая $\rho_{F}=75 \mu \mathrm{Ohm} \mathrm{cm} / \mathrm{at} . \%$ [2]. Концентрации дефектов в сплаве Fe13,6Cr-1,9Si при $120 \mathrm{~K}$ могут быть оценены только грубо как 60, 45 и 38 appm, так как удельный вклад ДФ в ОЭС в этом сплаве сильно отличается от известного значения [2] в нелегированных сплавах. Концентрации $60 \mathrm{appm}$ в обоих сплавах достигаются при облучении дозой $\sim 6,6 \times 10^{17}$ эл/ $\mathrm{cm}^{2}$.

ОЭС сплава $\mathrm{Fe} 13,6 \mathrm{Cr}-1,9 \mathrm{Si}\left(\rho_{0}=55 \pm 3 \mu \mathrm{Ohm} \mathrm{cm}\right)$ в 2,5 раза превышает ОЭС сплава $\mathrm{Fe}-13,4 \mathrm{Cr}\left(\rho_{0}=22 \pm 1 \mu \mathrm{Ohm} \mathrm{cm}\right)^{4}$. Одновременно $\rho_{F}$ в сплаве $\mathrm{Fe} 13,6 \mathrm{Cr}-1,9 \mathrm{Si}$ примерно вдвое меньше чем в сплаве $\mathrm{Fe}-13,4 \mathrm{Cr}$. Вследствие этих факторов, а также более медленного отжига дефектов, погрешность определения скорости возврата ОЭС в сплаве $\mathrm{Fe} 13,6 \mathrm{Cr}-1,9 \mathrm{Si}$ при температурах ниже 300 К примерно на порядок превышает такую погрешность в сплаве $\mathrm{Fe}-13,4 \mathrm{Cr}$ и может достигать 30-40 \%. При таких погрешностях полученные спектры являются недостоверными. Поэтому для образцов сплава Fe13,6Cr-1,9Si спектры возврата ОЭС приводятся только начиная с $290 \mathrm{~K}$.

\section{3. Результаты и обсуждение}

Данные по возврату ОЭС представлены на рис. 1. Зависимости $R(T)$ для образцов одного и того же сплава, облученных до разных концентраций ДФ, совпадают при низких температурах и начинают расходиться выше 200 К для сплава $\mathrm{Fe}-13,4 \mathrm{Cr}$ и $~ 300 \mathrm{~K}$ для $\mathrm{Fe} 13,6 \mathrm{Cr}-1,9 \mathrm{Si}$.

\footnotetext{
${ }^{4}$ Вклад кремния в удельное ОЭС сплава $\mathrm{Fe}-16 \mathrm{Cr}$ составляет около $17 \mu \mathrm{Ohm} \mathrm{cm} / \mathrm{at} . \%$ [1].
} 
Наблюдаемое расхождение кривых возврата ОЭС, полученных для различных исходных концентраций дефектов типично для систем, в которых при отжиге после низкотемпературного облучения происходит формирование ближнего порядка или, другими словами, ближнее упорядочение (БУ), ускоренное миграцией радиационных дефектов [6].

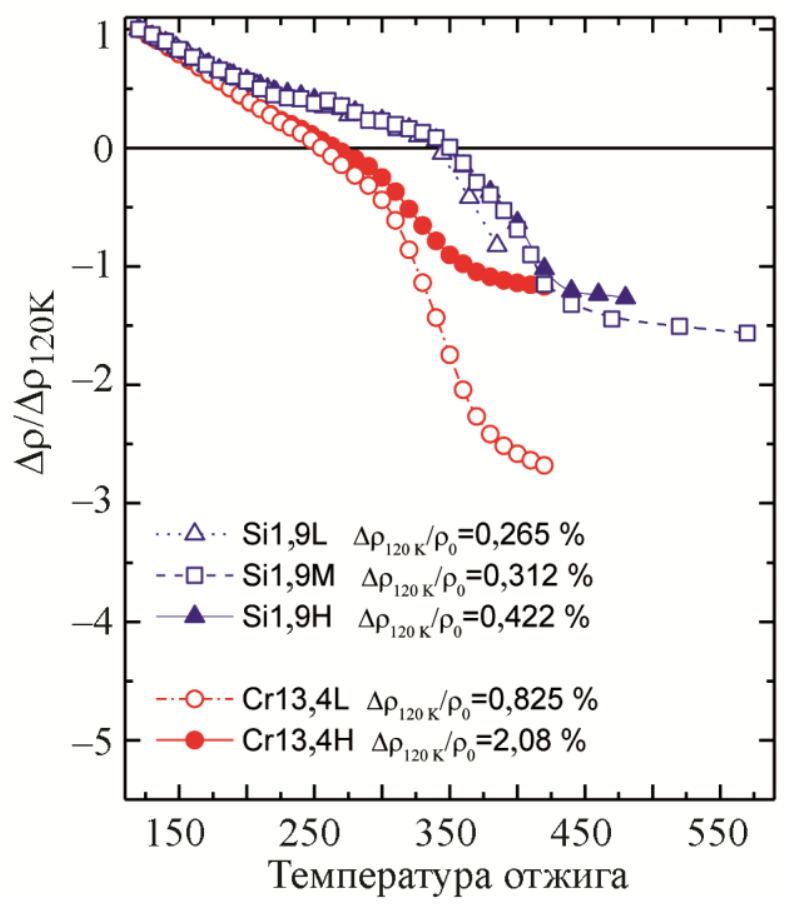

Рис. 1. Кривые возврата ОЭС образцов сплавов $\mathrm{Fe}-13,4 \mathrm{Cr}$ и $\mathrm{Fe} 13,6 \mathrm{Cr}-1,9 \mathrm{Si}$, облученных до разных значений приростов ОЭС. Погрешности измерений меньше размеров символов

В системах с БУ $R(T)$ состоит из двух частей, обусловленных присутствием дефектов и БУ, т. е. $R(T)=R_{d}(T)+R_{S R O}(T)=\Delta \rho_{d}(T) / \Delta \rho_{i r r}+\Delta \rho_{S R O}(T) / \Delta \rho_{i r r}$, где $\Delta \rho_{d}(T)$ и $\Delta \rho_{S R O}(T)$ являются текущими приростами ОЭС, обусловленными присутствием дефектов и БУ ${ }^{5}$, соответственно, после ступенчатого отжига вплоть до температуры $T$ включительно. Известно [8], что $\Delta \rho_{S R O}$ является функцией числа прыжков дефектов и становится заметным, когда общее число прыжков всех дефектов (СМА или вакансий) $N_{d}$ достигает величины порядка единицы на атом. Такое количество прыжков достигается только при дальней миграции дефектов. При взаимодействии дефектов из одной пары Френкеля, т.е. при рекомбинации близких пар и коррелированной миграции или захвате дефектов на ловушках, концентрация которых существенно превышает концентрацию дефектов (это наш случай с захватом дефектов на кремневых агрегатах), пробег дефектов (число прыжков) достаточно мал и вклад от БУ в ОЭС является пренебрежимо малым.

Физический смысл расхождения можно понять из следующих рассуждений [3]. Изменение в $\Delta \rho_{S R O}(T)$ при отжиге на каждой $i$-й ступени $\left(\Delta \rho_{S R O}\left(T_{i}\right)-\Delta \rho_{S R O}\left(T_{i-1}\right)\right)$ достаточно мало и с хорошей точностью прямо пропорционально $N_{d}\left(T_{i}\right)-N_{d}\left(T_{i-1}\right)$, т.е. числу прыжков дефектов при отжиге на этой ступени, где $N_{d}\left(T_{i}\right)$ общее число прыжков после отжига при $T_{i}$. При нормировке $\Delta \rho_{S R O}\left(T_{i}\right)-\Delta \rho_{S R O}\left(T_{i-1}\right)$ на $\Delta \rho_{i r}$ (что пропорционально исходной концентра-

\footnotetext{
${ }^{5}$ Величина $\Delta \rho_{S R O}(T)$ может быть как положительной, так и отрицательной в отличие от $\Delta \rho_{d}$, величина которого является положительной. Индекс SRO от английского Short-Range Order.
} 
ции дефектов $\left.C_{F}^{0}\right)$ получаемая разность $\Delta R_{S R O}\left(T_{i}\right)=R_{S R O}\left(T_{i}\right)-R_{S R O}\left(T_{i-1}\right)$ становится пропорциональной $\left[N_{d}\left(T_{i}\right)-N_{d}\left(T_{i-1}\right)\right] / C_{F}^{0}$, т.е. среднему числу прыжков на данной ступени, приходящемуся на один исходный дефект. Так как в течение отжига сохраняется пропорциональность между текущей и исходной концентрациями дефектов, то величина $\Delta R_{S R O}\left(T_{i}\right)$ оказывается также пропорциональной среднему числу прыжков одного дефекта на данной ступени отжига, т. е. $\Delta R_{S R O}\left(T_{i}\right) \sim\left[n_{d}\left(T_{i}\right)-n_{d}\left(T_{i-1}\right)\right]$.

Известно, что в процессе отжига после низкотемпературного облучения дефекты взаимодействуют преимущественно друг с другом. Поэтому средний пробег (среднее число прыжков) одного дефекта на каждой ступени отжига определяется вероятностью его встречи с другими дефектами, которая обратно пропорциональна как текущей, так и исходной концентрациям дефектов. Для наших рассуждений важна пропорциональность исходной концентрации дефектов, т. е. $\Delta R_{S R O}\left(T_{i}\right) \sim\left[n_{d}\left(T_{i}\right)-n_{d}\left(T_{i-1}\right)\right] \sim 1 / C_{d}^{0}$. Отсюда следует, что абсолютная величина $\left|\Delta R_{S R O}\left(T_{i}\right)\right|$ уменьшается с увеличением исходной концентрации дефектов. Если рассматривать два одинаковых образца с разной концентрацией дефектов - высокой ( $H$ ) и низкой $(L)$, то всегда справедливо соотношение $\left|\Delta R_{S R O}\left(T_{i}\right)\right|^{L}>\left|\Delta R_{S R O}\left(T_{i}\right)\right|^{H}$.

Величина $R_{d}(T)$ слабо меняется при относительно небольших (до $\sim 4$ раз) изменениях концентрации дефектов (относительные различия, как правило, не превышают $10 \%$ во всем температурном диапазоне отжига дефектов). Поэтому зависимости $R_{d}(T)$ для одинаковых образцов с разной концентрацией дефектов отличаются слабо.

Отжиг дефектов в образцах с разной концентрацией дефектов до начала дальней миграции протекает идентично. $R(T)$ состоит из одной компоненты $R_{d}(T)$, так как $R_{S R O}(T)=0$ и кривые $R^{H}(T)$ и $R^{L}(T)$ совпадают. Когда начинается дальняя миграция дефектов, то $R_{S R O}(T)$ становится отличным от нуля, но $R_{S R O}^{H}(T) \neq R_{S R O}^{L}(T)$.

Величина $R_{S R O}(T)$ после отжига на ступени при данной температуре $T$ есть сумма всех $\Delta R_{S R O}\left(T_{i}\right)$ на предшествующих ступенях отжига, включая $\Delta R_{S R O}(T), \quad$ т. е. $R_{S R O}(T)=\sum_{i=1}^{T_{i}=T} \Delta R_{S R O}\left(T_{i}\right)$ и на каждой ступени $\left|\Delta R_{S R O}^{L}\left(T_{i}\right)\right|>\left|\Delta R_{S R O}^{H}\left(T_{i}\right)\right|$. В результате $\left|R_{S R O}^{L}(T)\right|>$ $\left|R_{S R O}^{H}(T)\right|$ и разница между $\left|R_{S R O}^{L}(T)\right|$ и $\left|R_{S R O}^{H}(T)\right|$ растет с увеличением температуры.

Другими словами, зависимости $R_{S R O}^{H}(T)$ и $R_{S R O}^{L}(T)$ расходятся с ростом температуры отжига. Это расхождение приводит к расхождению кривых $R^{H}(T)$ и $R^{L}(T)$, т.к. компоненты $R_{d}^{L}(T)$ и $R_{d}^{H}(T)$ различаются слабо. Это означает, что появление в возврате ОЭС компоненты $R_{S R O}(T)$, когда последняя становится отличной от нуля, ведет к расхождению кривых возврата $R^{L}(T)$ и $R^{H}(T)$.

Таким образом, расхождение кривых возврата указывает как на начало дальней миграции дефектов, так и на начало БУ, ускоренного дальней миграцией дефектов (рис. 1). Температура начала расхождения соответствует низкотемпературному краю стадии начала дальней миграции наиболее подвижных дефектов из пары Френкеля. В концентрированных сплавах это могут быть как СМА [6] (аналогично случаю чистых металлов), так и вакансии $[2,9]$ (в отличие от случая чистых металлов).

$\mathrm{B}$ сплавах $\mathrm{Fe}-\mathrm{Cr}$ с концентрацией $\mathrm{Cr}$ выше 10 ат. \% при термическом отжиге и отжиге после низкотемпературного облучения происходит БУ по типу ближнего расслоения, кото- 
рое проявляется, соответственно, как уменьшение ОЭС [10] и падение $R(T)$ ниже нуля [7, 11-13]. На рис. 1 видно, что вскоре после начала расхождения кривых $R(T)$ последнее падает ниже нуля. Это указывает на протекание процесса БУ по типу расслоения в наших сплавах, которое является причиной расхождения кривых $R(T)$.

Наиболее точно температура начала расхождения кривых $R(T)$ определяется на спектрах возврата ОЭС (рис. 2). Она равна 202 К для сплава $\mathrm{Fe}-13,4 \mathrm{Cr}$ (рис. 2, вставка) и $330 \mathrm{~K}$ для сплава $\mathrm{Fe} 13.6 \mathrm{Cr}-1,9 \mathrm{Si}$. Эти температуры соответствуют низкотемпературному краю стадий начала дальней миграции, пики которых располагаются при $220 \mathrm{~K}$ для Fe-13,4Cr (рис. 2, вставка) и при $370 \mathrm{~K}$ для Fe13,6Cr-1,9Si. Ниже 330 K в сплаве Fe13,6Cr-1,9Si существуют только одиночные (не входящие в состав кластеров дефектов) вакансии и СМА. В сплаве $\mathrm{Fe}-13,4 \mathrm{Cr}$, согласно [5], вакансии захватываются и удерживаются атомами углерода до $320 \mathrm{~K}$, там, где на рис. 2 наблюдается пик большой стадии возврата ОЭС, связанной с освобождением вакансий. Из-за захвата на атомах углерода, концентрация которых намного превышает концентрации дефектов, миграция вакансий ниже $320 \mathrm{~K}$ не влияет на БУ в сплаве $\mathrm{Fe}-13,4 \mathrm{Cr}$. Поэтому расхождение кривых $R(T)$ выше $202 \mathrm{~K}$ и стадия возврата ОЭС при $220 \mathrm{~K}$ обусловлены началом дальней миграции СМА (рис. 2, вставка).

Спектр сплава Fe13,6Cr-1,9Si сильно отличается от спектра $\mathrm{Fe}-13,4 \mathrm{Cr}$ (рис. 2). Дальняя миграция дефектов в Fe13,6Cr-1,9Si начинается при температурах, когда оба компонента пары Френкеля уже подвижны в $\mathrm{Fe}-13,4 \mathrm{Cr}$. Это указывает на то, что температура начала дальней миграции ДФ в Fe13,6Cr-1,9Si определяется их взаимодействием только с атомами $\mathrm{Si}$. Это логично, поскольку концентрация кремния превышает концентрацию углерода и других примесей, по крайней мере в сорок раз.

$\mathrm{B}$ чистом сплаве $\mathrm{Fe}-9 \mathrm{Cr}$ [2] пик стадии начала миграции вакансий и низкотемпературный край этой стадии наблюдаются при 205 К и 175 К соответственно. Поэтому дальняя миграции дефектов в отсутствии примеси углерода начинается при 175 К и сдвиг начала дальней миграции при легировании 2 ат. \% Si до 330 К необходимо вести от 175 К.

Процент возврата в сплаве Fe13,6Cr-1,9Si после отжига при 330 К (к началу дальней миграции) составляет $\sim 13$ или $\sim 7 \%$, если нормировка прироста ОЭС осуществляется после облучения при низкой температуре (4,2-21 К). Ранее [2] упоминалось, что процент возврата ОЭС к началу дальней миграции в нелегированном сплаве $\mathrm{Fe}-9 \mathrm{Cr}$ составляет 18 \% (при нормировке при $21 \mathrm{~K})$. Разница в процентах возврата к началу дальней миграции указывает на захват ДФ на атомах $\mathrm{Si}$, который сопровождается потерей удельного вклада дефектов в ОЭС. Известно, что захват вакансий на атомах кремния в $\mathrm{Fe}$ [14] приводит к уменьшению вклада вакансий на 40 \% от вклада пары Френкеля. В нашем случае уменьшение вклада составляет $60 \%$. Более высокий процент потери вклада в ОЭС может свидетельствовать о захвате вакансий на нескольких атомах кремния (кремниевых агломератах). Подобный эффект (увеличение потери удельного вклада дефектов в ОЭС) известен, когда вакансии в Fе перезахватываются с одного на два атома углерода [15].

В спектрах возврата ОЭС образцов сплава $\mathrm{Fe} 13,6 \mathrm{Cr}-1,9 \mathrm{Si}$ в температурном интервале 330-480 К при 370 и 420 К наблюдаются два пика стадий возврата. Возврат ОЭС составляет $13 \%$ при 330 К и падает до - 127 и -146 \% (существенно ниже исходного уровня) при 480 К. Отсюда следует, что более $90 \%$ возврата ОЭС в данном температурном интервале обусловлено процессами БУ, ускоренного дальней миграцией дефектов, т. е. $R(T) \approx R_{S R O}(T)$. 


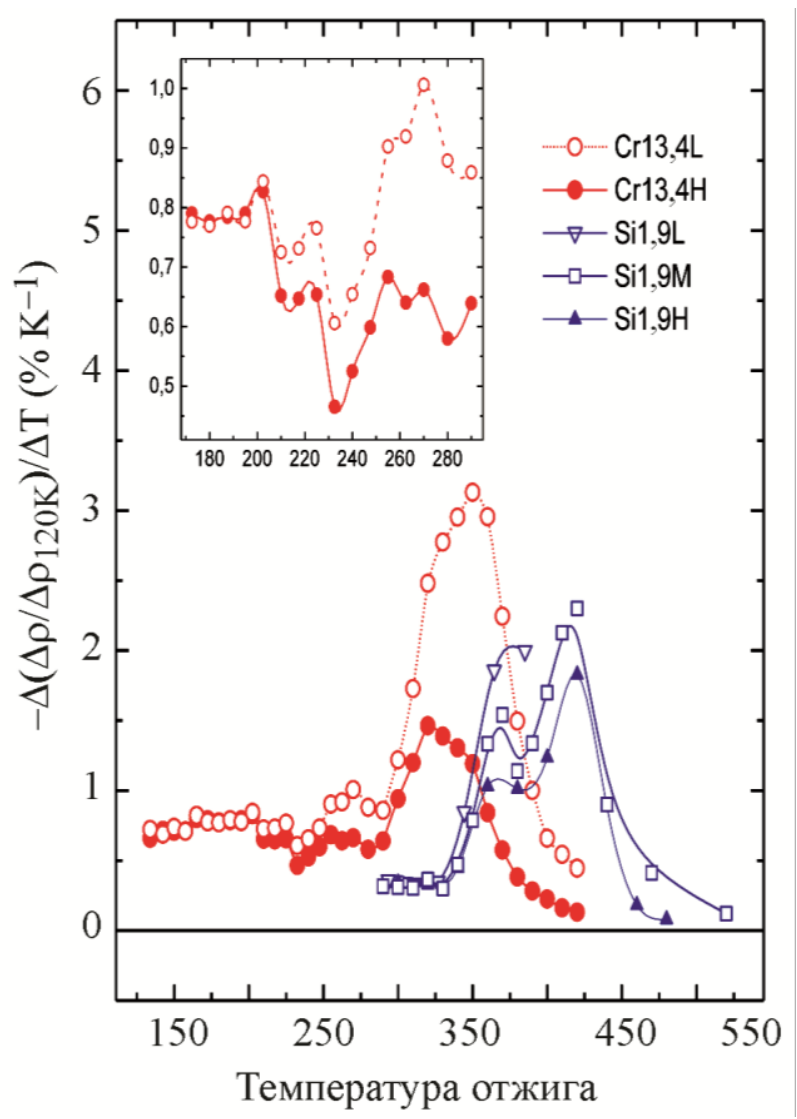

Рис. 2. Спектры возврата ОЭС для образцов сплавов Fe-Cr (рис. 1). Вставка: подробное изображение спектров возврата ОЭС для образцов сплава $\mathrm{Fe}-13,4 \mathrm{Cr}$ вблизи температуры начала расхождения кривых возврата. Погрешности измерения примерно соответствуют размерам символов. Для образцов сплава $\mathrm{Fe}-13,4 \mathrm{Cr}$ погрешности соответствуют размеру символов на вставке

Ранее [4] мы выделяли в возврате ОЭС $R(T)$ сплава $\mathrm{Fe}-4 \mathrm{Cr}$ часть, обусловленную БУ $R_{S R O}(T)$. В этой части видны только две стадии, связанные с началом дальней миграции CMА и вакансий. Это вызвано тем, что другие процессы, происходящие при отжиге дефектов, слабо влияют на БУ. Поэтому две стадии возврата, наблюдаемые в $R_{S R O}(T)$ сплава $\mathrm{Fe} 13.6 \mathrm{Cr}-1,9 \mathrm{Si}$, напрямую связаны с началом дальней миграции СМА и вакансий.

Способность СМА ускорять БУ меньше, чем соответствующая способность вакансий. По этой причине в сплаве $\mathrm{Fe}-4 \mathrm{Cr}$ [4] пик стадии в $R_{S R O}(T)$, связанной с началом дальней миграции СМА, имеет меньшую амплитуду, чем соответствующий пик стадии, связанной с началом дальней миграции вакансий. По аналогии пик стадии с более низкой амплитудой при 370 К в Fe13,6Cr-1,9Si должен интерпретироваться как начало дальней миграции СMA, a более высокий пик при $420 \mathrm{~K}$ - как начало дальней миграции вакансий.

Таким образом, с учетом результатов и их анализа видно, что данные по возврату ОЭС в сплавах $\mathrm{Fe}-13,4 \mathrm{Cr}$ и $\mathrm{Fe} 13,6 \mathrm{Cr}-1,9 \mathrm{Si}$ подтверждают данные, полученные методом аннигиляции позитронов в сплавах $\mathrm{Fe} 16 \mathrm{Cr}-\mathrm{Si}$ [1], о захвате CMA и вакансий и удержании их до достаточно высоких температур при наличии в сплаве 1,5-2 ат. \% Si за счет формирования кремниевых агрегатов. С одной стороны, иммобилизация радиационных дефектов может усиливать их взаимную рекомбинацию и за счет этого уменьшать радиационную повреждаемость. С другой стороны, если агрегаты имеют тенденцию к заметному росту под облучением, то это может приводить к охрупчиванию. Формирование кремниевых агрегатов 
не будет оказывать отрицательного влияния на свойства материала только в том случае, если агрегаты остаются достаточно малыми (2-3 атома).

\section{4. Выводы}

Легирование сплава $\mathrm{Fe}-\mathrm{Cr} 2$ ат. \% Si сдвигает начало дальней миграции радиационных дефектов со 175 до $330 \mathrm{~K}$.

Стадии начала дальней миграции СMA и вакансий в сплаве Fe13,6Cr-1,9Si сдвигаются до 370 и 420 К по сравнению с 220 и 205 К в чистых сплавах Fe-Cr.

\section{Благодарность}

Работа выполнена в рамках государственного задания МИНОБРНАУКИ России (тема «Функиия», № АААА-А19-119012990095-0). Автор выражает благодарность Д. А. Перминову за критические замечания при чтении работы.

\section{Литература}

1. Druzhkov A. P., Nikolaev A. L. Effect of Si concentration on formation of vacancy complexes in electron-irradiated Fe16Cr-Si alloys studied by positron annihilation // J. Nucl. Mater. 2018. - Vol. 508. - P. 100-106. - DOI: 10.1016/j.jnucmat.2018.05.040.

2. Nikolaev A. L. Specificity of stage III in electron-irradiated Fe-Cr alloys // Phil. Mag. 2007. - Vol. 87, No. 31. - P. 4847-4874. - DOI: 10.1080/14786430701468977.

3. Nikolaev A. L. Difference approach to the analysis of resistivity recovery data for irradiated short-range ordered alloys // Phil. Mag. - 2009. - Vol. 89, No. 12. - P. 1017-1033. DOI: 10.1080/14786430902835651.

4. Nikolaev A. L. Recovery of electrical resistivity, short-range order formation and migration of defects in electron-irradiated Fe-4Cr alloy doped with carbon // Phil. Mag. - 2011. - Vol. 91, No. 6. - P. 879-898. - DOI: 10.1080/14786435.2010.534740.

5. Druzhkov A. P., Nikolaev A. L. Effects of solute atoms on evolution of vacancy defects in electron-irradiated Fe-Cr-based alloys // J. Nucl. Mater. - 2011. - Vol. 408. - P. 194-200. DOI: 10.1016/j.jnucmat.2010.11.036.

6. Dimitrov C., Dimitrov O. Composition dependence of defect properties in electronirradiated Fe-Cr-Ni solid solutions // J. Phys. F: Met. Phys. - 1984. - Vol. 14. - P. 793-811. DOI: 10.1088/0305-4608/14/4/005.

7. Benkaddour A., Dimitrov C., Dimitrov O. Irradiation-induced defects in ferritic Fe-Cr alloys // Mater. Sci. Forum. - 1987. - Vol. 15-18. - P. 1263-1268. - DOI: 10.4028/www.scientific.net/MSF.1518.1263.

8. Investigation of the kinetics of short-range order formation and quenched-in vacancy annihilation in Au-15 at. \% Ag by resistivity measurements / W. Kohl, R. Scheffel., H. Heidsiek, K. Lucke // Acta metall. - 1983. - Vol. 31, No 11. - P. 1895-1908. - DOI: 10.4028/www.scientific.net/MSF.15-18.1263.

9. Vaessen P., Lengeler B., Shilling W. Recovery of electrical resistivity in electronirradiated concentrated silver-zinc alloys // Rad. Effects - 1984. - Vol. 81. - P. 277-292. DOI: $10.1080 / 00337578408206075$.

10. Mirebeau I., Hennion M., Parette G. First measurement of short-range-order inversion as a function of concentration in a transition alloy // Phys. Rev. Lett. - 1984. - Vol. 53. - P. 687-690. DOI: 10.1103/PhysRevLett.53.687.

11. The influence of carbon on the resistivity recovery of proton irradiated $\mathrm{Fe}-11$ at.\% $\mathrm{Cr}$ alloys / G. Apostolopoulos, V. Lukianova, Z. Kotsina, A. Lagoyannis, K. Mergia, S. Harissopoulos, S. Messoloras // Nuclear Materials and Energy - 2016.- Vol. 9. - P. 465-470. DOI: 10.1016/j.nme.2016.09.007. 
12. Gómez-Ferrer Begoña. Resistivity recovery in $\mathrm{Fe}$ and $\mathrm{FeCr}$ alloys. - SpringerBriefs in Applied Sciences and Technology, 2016.

13. Nikolaev A. L., Arbuzov V. L., Davletshin A. E. On the effect of impurities on resistivity recovery, short-range ordering, and defect migration in electron-irradiated concentrated $\mathrm{Fe}-\mathrm{Cr}$ alloys // J. Phys.: Condens. Matter. - 1997. - Vol. 9. - P. 4385-4402. - DOI: 10.1088/09538984/9/21/006.

14. Interstitial migration in dilute $\mathrm{Fe}-\mathrm{Si}$ and $\mathrm{Fe}-\mathrm{Au}$ alloys / F. Maury, A. Lucasson, P. Lucasson, P. Moser, Y. Loreaux // J. Phys. F: Met. Phys. - 1985. - Vol. 15. - P. 1465-1484. DOI:10.1088/0305-4608/15/7/007.

15. The resistivity recovery of high purity and carbon doped iron following low temperature electron irradiation / S. Takaki, J. Fuss, H. Kugler, U. Dedek, H. Schultz // Rad. Effects - 1983. Vol. 79. - P. 87-122. - DOI: 10.1080/00337578308207398. 